\title{
Experimental analysis of lateral impact on planar brittle material
}

\author{
F. P. M. dos Santos, ${ }^{1,2, *}$ V. C. Barbosa, ${ }^{1, \dagger}$ R. Donangelo, ${ }^{1,3, \ddagger}$ and S. R. Souza ${ }^{1,4, \S}$ \\ ${ }_{1}^{1}$ Instituto de Física, Universidade Federal do Rio de Janeiro, Cidade Universitária, CP 68528, Rio de Janeiro, RJ 21941-972, Brazil \\ ${ }^{2}$ CEFET Química de Nilópolis, Av. Lúcio Tavares 237, Nilópolis, RJ 26530-060, Brazil \\ ${ }^{3}$ Facultad de Ingeniería, Instituto de Física, CP 30, 1100 Montevideo, Uruguay \\ ${ }^{4}$ Instituto de Física, Universidade Federal do Rio Grande do Sul, Av. Bento Gonçalves 9500, \\ CP 15051, Porto Alegre, RS 91501-970, Brazil
}

(Received 23 November 2009; revised manuscript received 25 March 2010; published 20 April 2010)

\begin{abstract}
The fragmentation of alumina and glass plates due to lateral impact is studied. A few hundred plates have been fragmented at different impact velocities and the produced fragments are analyzed. The method employed in this work allows one to investigate some geometrical properties of the fragments, besides the traditional size distribution usually studied in former experiments. We found that, although both materials exhibit qualitative similar fragment size distribution function, their geometrical properties appear to be quite different. A schematic model for two-dimensional fragmentation is also presented and its predictions are compared to our experimental results. The comparison suggests that the analysis of the fragments' geometrical properties constitutes a more stringent test of the theoretical models' assumptions than the size distribution.
\end{abstract}

DOI: 10.1103/PhysRevE.81.046108

PACS number(s): 46.50.+a, 62.20.M-

\section{INTRODUCTION}

The fragmentation process is a common phenomenon that is found both in natural [1-7] and industrial processes [8-11], and takes place on scales ranging from the collision of galaxies [1] and asteroids [2] to the breakup of heavy nuclei [6]. The underlying physics of the fragmentation phenomenon is very different for macroscopic and microscopic systems, since quantum effects have to be considered in the latter, and also because the mechanisms that lead to their breakup are not the same. In this work, we focus on the macroscopic systems. From both the economic and academic points of view, it is important to understand the mechanisms that govern this process and, based on them, build models that allow predictions for different scenarios.

As discussed in Ref. [12], brittle materials such as glasses and ceramics exhibit no macroscopic plastic deformation as they are subject to tensile loading capable of producing a stress level greater than their limiting elastic limit. Thus, once this stress threshold is reached, the fragmentation process immediately starts. The first systematic studies of brittle fracture using statistical arguments were performed about 60 years ago by Weibull [13], who introduced a probability distribution function (named Weibull distribution) to describe the fragment size distributions.

Recently, this subject has regained a great deal of attention since Oddershede et al. [14] showed that the size $(s)$ distribution of the fragments produced in the breakup of brittle objects is given by a power law $s^{-\beta}$ which is fairly independent of the specific material employed. It is essentially determined by the morphology of the fragmenting object and the power law exponent $\beta=1.63$ was found for

\footnotetext{
*filipepms@gmail.com

†valmar@if.ufrj.br

†rdonangelo@gmail.com

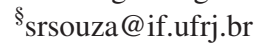

spherical gypsum balls, $\beta=1.08$ for thin gypsum disks, and $\beta=1.05$ for gypsum rods. These authors showed that this scaling law is observed for fragments whose dimensions are smaller than the smallest dimension of the original object [14]. Subsequent investigations made in Ref. [15], where glass rods were dropped from increasing heights, suggest that the power law exponent rises from $\beta \approx 1.2$ to 1.5 as the violence of the impact increases (larger heights). A similar experiment has been reported in Ref. [16], where a sandwich of thin glass or plaster plates, inserted between stainless steel plates, was hit by an iron projectile, which was dropped on the target at normal angles to its surface. The fragment distribution is also given by a power law whose exponent increases from $\beta=1.5$ to 1.7 according to the violence of the impact. Although these studies agree qualitatively, the observed power law exponents are somewhat different. Qualitatively different results were obtained in Ref. [17] where the mass distribution of fragments produced in the fragmentation of thick plates of dry clay were described by two power laws of exponents $\beta_{S}=1.5-1.7$ for small fragments and $\beta_{L}$ $=1.1-1.2$ for the larger ones. These authors suggest that the exponents are associated with the dimensionality of the fragmenting object and the length of the fragments. They also investigated the problem using models based on very different pictures for the fragmentation process and found that both lead to the same mass distributions. This finding strongly suggests that the study of other observables, besides the mass/size distribution, is necessary to unveil the underlying physics of the fragmentation process. This conclusion is one of the motivations for the present work.

Other recent experimental studies investigated the properties of fragmentation in different scenarios. For instance, the breakup of closed thin shells due to impact and also as a result of the explosion of a combustible mixture has been studied in Ref. [18]. For practical reasons, eggs have been used in these experiments. The authors found that the mass distribution follows a power law, in both cases, whose exponent $\beta=1.35 \pm 0.02$ lies between the values $\beta_{L}$ and $\beta_{S}$ observed in Ref. [17] and mentioned above. On the other hand, 
studies on the fragmentation of mercury drops $[19,20]$, due to the fall from a fixed height on a hard surface, show that the fragment size distribution is fairly well described by a power law of exponent $\beta \approx 1.1$ over a wide range of sizes [19].

The role played by the constraints imposed to the fragmenting system has been investigated in Ref. [21], where slender brittle rods, made up of dry spaghetti (besides other brittle materials), have been kept fixed at one end and axially impacted at the other. The corresponding mass distribution exhibits bumps around $\lambda / 2$ and $\lambda / 4$, where $\lambda$ is the preferred wavelength for the buckling instability. This issue was, to some extent, also investigated in Ref. [16] where the plaster plates were laterally bombarded by a high velocity projectile and $\beta$ was found to lie within 1.1 and 1.3 , which contrasts with the values mentioned above when the plates are hit on their surfaces. There have also been experimental studies that focused on the internal details of the fractures, such as those reported in Ref. [22], where the statistical distribution function for the height fluctuations along the fracture length was carefully examined and found to be Gaussian.

Owing to the great complexity of the fracture process [23-26], several schematic models have been proposed to describe the phenomenon (see [19,24-37] and references therein). Some of them try to incorporate microscopic information [25-28,37] whereas the majority of the approaches are minimalistic models which use as few parameters as possible to describe the fragmentation process [19,29-36]. Due to the very small time scale of the fracture dynamics [23], most of the experimental information is usually restricted to the final state properties of the system. Therefore, it is very difficult to single out the appropriate scenario from these models since most of them make predictions which agree reasonably well with the available experimental data.

In this work we present the results of an experiment from which we extract further observables associated with the fragments' geometry and size, besides their mass distribution. By providing more detailed information on the fragments produced in the breakup of brittle material, we intend to make it possible to distinguish between the different scenarios assumed in the theoretical models or, at least, to constrain the range of their free parameters. We study the fragmentation due to lateral impacts on thin plates of alumina and glass, so that our experiment is similar to one of the measurements made in Ref. [16], mentioned above. As described below, we have been particularly careful in controlling the impact velocities, in order to minimize effects associated with mixing the energy deposited into the system. Great care has also been taken in order to ensure that the plates are always hit along their lateral side, in order to minimize any bias due to angle mixing.

The remainder of this paper is organized as follows. Our experimental setup is described in detail in Sec. II and the model devised to interpret the experimental results is presented in Sec. III. The results are discussed in Sec. IV and the main conclusions are summarized in Sec. V.

\section{EXPERIMENTAL SETUP}

We have used 232 square plates $\left(10 \times 10 \mathrm{~cm}^{2}\right)$ to study the properties of the breakup of thin brittle plates due to
TABLE I. Properties of the square plates used in this work.

\begin{tabular}{lcccc}
\hline \hline Material & $\begin{array}{c}\text { Thickness } \\
(\mathrm{mm})\end{array}$ & $\begin{array}{c}\text { Sides } \\
(\mathrm{mm})\end{array}$ & $\begin{array}{c}\text { Density } \\
\left(\mathrm{g} / \mathrm{cm}^{3}\right)\end{array}$ & Quantity \\
\hline Alumina & 0.5 & 100.0 & 3.80 & 156 \\
Glass & 1.0 & 100.0 & 2.46 & 76 \\
\hline \hline
\end{tabular}

lateral impacts. Two different materials have been considered: alumina $(0.5 \mathrm{~mm}$ thickness $)$ and glass $(1.0 \mathrm{~mm}$ thickness). The samples have different microscopic structure, but both have noncrystalline atomic arrangement. The alumina targets were manufactured by Coorstek Inc. and the glass plates by Goes Vidros. None of the plates has been previously sintered. Their physical properties, as well as the number of analyzed plates, are given in Table I.

A plate is laid down on a flat surface and it is hit laterally by a piston. No constraints are imposed on the plate, so that it can move freely when hit. A schematic illustration of the apparatus is shown in Fig. 1. It consists of a compressed air cylinder which is connected to a reduction pressure valve. The latter allows one to suitably fix the pressure in the pneumatic cylinder connected at its end. In order to ensure uniform impacts along the plate's length, a $12 \times 5 \times 2 \mathrm{~cm}^{3}$ (length $\times$ height $\times$ width) steel block is attached to the external end of the piston rod. Since the length of this block $(12 \mathrm{~cm})$ is larger than that of the plates $(10 \mathrm{~cm})$, which are placed with a side parallel to the block, the impact is very homogeneous. In order to investigate this issue, we have also checked that when a thin aluminum plate (whose size is similar to that of the plates) is hit by the piston, no particular spots on its lateral side are observed. The basis for the targets is fixed on an iron plate. To it is attached a polystyrene shock absorber bar, which is intended to stop the pneumatic cylinder before it reaches the end of the piston stroke, thus preserving the physical integrity of the apparatus. The compressed air is injected into the pneumatic cylinder through the activation of a high-speed electrical valve, allowing the steel block to accelerate and reach the target plate, before being stopped by the polystyrene stop. The piston touches the absorber bar only after the plate has been hit and ejected from the surface, so that the stopping system does not affect the impact velocity.

The velocity of the block impinging on the target is measured using two photodiodes located $1.2 \mathrm{~cm}$ apart from each

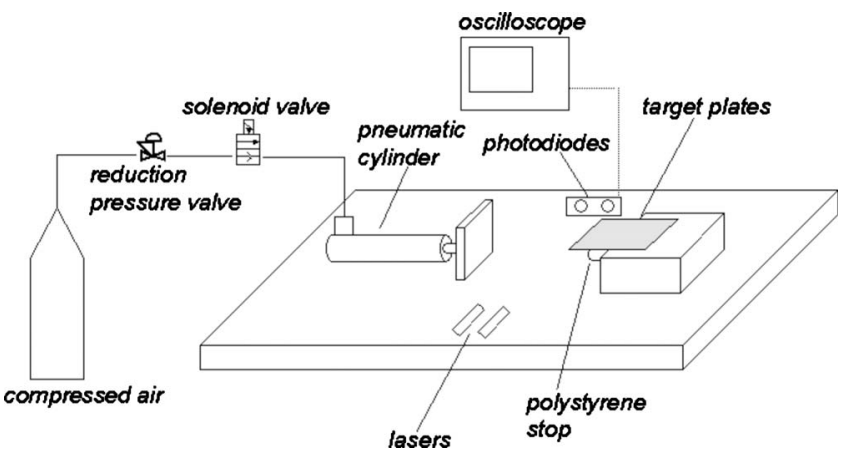

FIG. 1. Schematic illustration of the apparatus used in this work to study the fragmentation of brittle plates. For details, see the text. 


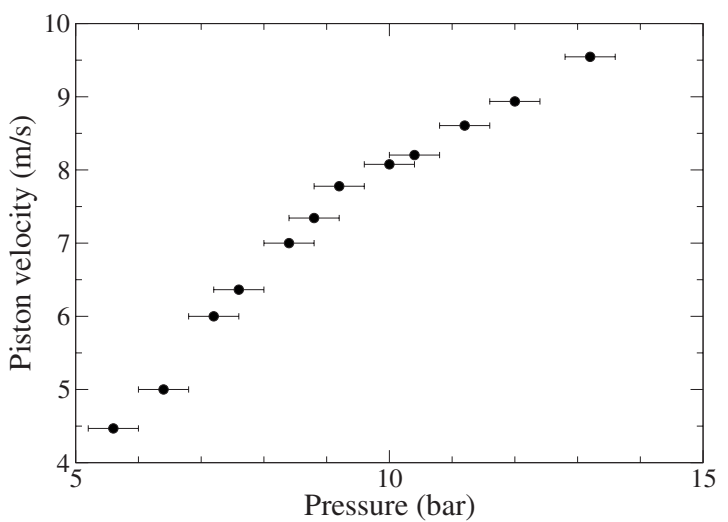

FIG. 2. Relation between the applied pressure and the velocity of the collision block.

other. Each of them is illuminated by a laser. As the light on either of the photodiodes is interrupted by the passage of the block, signals are transmitted to a digital oscilloscope, thus allowing the measurement of the block speed as a function of the applied pressure. The calibration curve obtained with the apparatus is presented in Fig. 2.

The minimal velocity necessary to break the samples depends on the material used as target. For our experimental setup, the threshold for the alumina plates is approximately $8.1 \mathrm{~m} / \mathrm{s}$ and it is $6.4 \mathrm{~m} / \mathrm{s}$ for the glass ones. Below these values, some of the plates occasionally break, but produce very few fragments. If a plate does not break, it is not used again as it may have suffered internal damages.

Since the targets are not fixed, they move freely after having been hit by the piston. In order to confine the produced fragments and at the same time preventing secondary fragmentation due to further impacts with the surroundings, we have placed the system inside a soft plastic enclosure with a silk bag at its right end. This part of the apparatus is not depicted in Fig. 1.

The produced fragments are placed on a high resolution scanner. This provides detailed images from which one determines their geometric properties. We have been particularly careful in placing the fragments on the scanner so as to prevent them from touching each other. For the alumina plates, we found that black and white images of $600 \mathrm{dpi}$ resolution are suitable for our purposes as they allow one to identify fragments whose sizes are on the order of $A_{\text {cut }}$ $=0.18 \mathrm{~mm}^{2}$, i.e., $A_{\text {cut }}=1.80 \times 10^{-5}$ smaller than the original objects $\left(A_{0}\right)$. The glass plates were scanned as 256 color bitmaps at 200 dpi resolution. Therefore, the smallest fragment's area studied in this case corresponds to $A_{\text {cut }}$ $=1.61 \mathrm{~mm}^{2}$. The fragments are identified by simply counting the contiguous active pixels on the scanned image, similarly to what is done in standard cluster recognition algorithms used in percolation theory. We stress that the analysis performed in this work provides much more information than the traditional ones which focus on the fragments' masses.

\section{SCHEMATIC MODEL FOR PLAQUE FRAGMENTATION}

Before presenting the fragmentation data obtained with the apparatus described above, we introduce a schematic

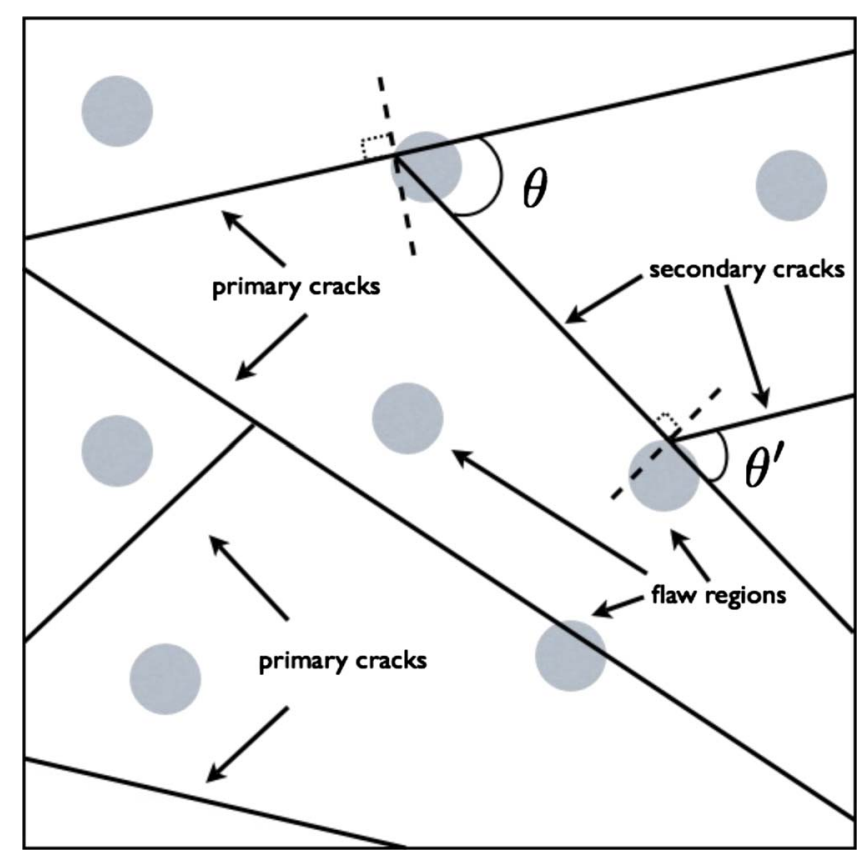

FIG. 3. (Color online) Illustration of the propagation of the cracks in the framework of the schematic fragmentation model introduced in this work. The initial cracks start at the left side of the plate. The definition of the bifurcation angle $\theta$ is also depicted in this figure. For details, see the text.

fragmentation model which will be of help in the interpretation of our experimental results.

As mentioned in the previous section, many models have been developed to describe fragmentation phenomena in different scenarios [19,24-36]. The confrontation of these models with the experimental results presented below is important in order to clarify the essential physical ingredients involved in the fragmentation process. However, due to the large number of models available in the literature, this is beyond the scope of the present work. We therefore devised a schematic two-dimensional Monte Carlo model, depicted in Fig. 3, inspired in the beautiful experiment reported by $\mathrm{Xu}$ and Rosakis [23], which we apply to interpret our experimental results. Although we keep it as simple as possible, we introduce the main ingredients we consider relevant to the process:

(i) At the beginning of the process, cracks are created at one of the lateral sides of the square plates of area $A_{0}=1$ and their propagation directions are randomly selected. This number $N_{c}$ of initial cracks is closely related to the violence of the impact and is a parameter of our model;

(ii) The propagation of the cracks start simultaneously and any of them stops only if its course is interrupted by another crack or if one of the borders is reached. For simplicity, we assume that the propagation speed is the same for all cracks.

(iii) A number $N_{f}$ of flaw regions are randomly placed over the surface of the plate. The number $N_{f}$ is sampled from a Poisson distribution with an average value $\left\langle N_{f}\right\rangle$, which is a parameter of the model. The flaw regions are considered to be circles, all of radius $R$, which is another parameter of our model;

(iv) When a crack enters into a flaw region, a new branch is created with probability $P_{c}$. Its propagation direction is 


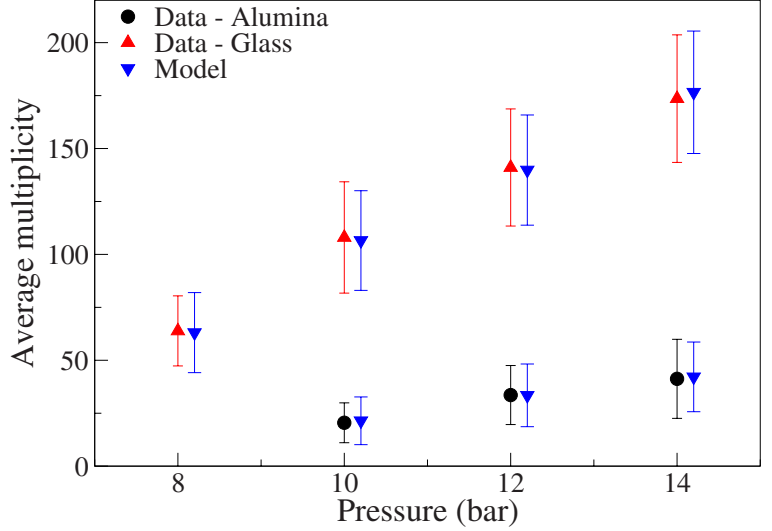

FIG. 4. (Color online) Fragment multiplicity as a function of the initial cylinder pressure.

uniformly sampled between $-\pi / 2$ and $+\pi / 2$, with respect to that of the initial crack. The latter, continues its course;

(v) In order to minimize the number of free parameters of the model, we argue intuitively that the number of initial cracks $N_{c}$ is closely related to the violence of the impact, as well as $P_{c}$. Although it is difficult to determine the relationship between $N_{c}$ and $P_{c}$, any reasonable function which gives $N_{c}=0$ for $P_{c} \rightarrow 0$ and leads to large values of $N_{c}$ for $P_{c} \rightarrow 1$, should provide equivalent qualitative results. For simplicity, we adopt $N_{c}=-10 \ln \left(1-P_{c}\right)$ throughout this work. Nevertheless, we have checked that, for instance, $N_{c}=10\left[\left(1-P_{c}\right)^{-1 / 2}\right.$ -1 ] leads to very similar results than the former expression if one employs slightly different $P_{c}$ values.

The average number of flaws $\left\langle N_{f}\right\rangle$ is a parameter of the model which is associated with the brittleness of the material. Therefore, different values of $\left\langle N_{f}\right\rangle$ and $R$ could be used for distinct materials. For simplicity, we fixed $\left\langle N_{f}\right\rangle=10000$ and $R=0.0005$ for both alumina and glass plates. (It should be noted that $A_{0}=1$ for the theoretical targets, which means that $\pi R^{2}$ should be scaled proportionally to $A_{0}$ if different values of $A_{0}$ are used.) Then, the probability of creating a new crack $P_{c}$ is the only parameter that we vary according to the violence of the impact and the material of the plates. Owing to the stochastic nature of the model, 10000 events are run for each case studied below.

\section{RESULTS}

The average fragment multiplicity is displayed in Fig. 4 as a function of the pressure for both alumina and glass plates. Since the experimental setup allows for small pressure variations, as represented by the error bars in Fig. 2, one should refer to $P$ as the average pressure. For brevity, in what follows, we denote it simply as pressure. As discussed in Sec. II, fragments of area smaller than $A_{\text {cut }}$ are not considered. For consistency, the same procedure is employed in the theoretical calculations. The model results, depicted by the upsidedown triangles, are slightly shifted to the right in order not to overlap with the data. All the error bars shown in this figure correspond to the standard deviation, i.e., they are associated with the width of the distribution. The model parameter $P_{c}$ was adjusted for each pressure used in the experiment. In the

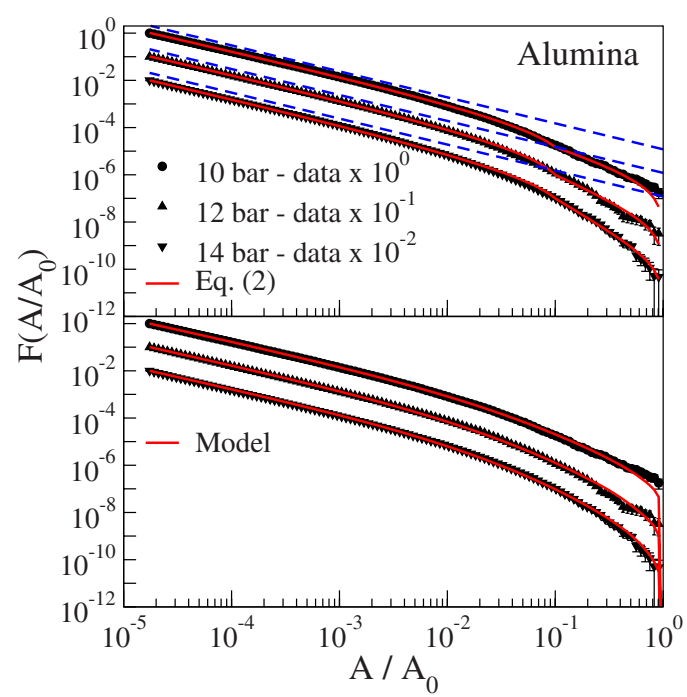

FIG. 5. (Color online) Area distribution for alumina plates at different pressures. The upper panel shows the power law fit (dashed lines) to the data with $\beta=1.1$ and the fit using Eq. (2) (full lines). The bottom panel displays the model results. For details, see the text.

case of the alumina plates, we used $P_{c}=0.329,0.393$ and 0.425 for $P=10,12$, and 14 bar, respectively. For the glass plates, we adopted $P_{c}=0.53,0.67,0.76$ and 0.85 for $P=8$, 10,12 , and 14 bar, also, respectively. This parameter set is used throughout this work, except where stated otherwise. These results show that the average fragment multiplicity increases steadily as a function of the pressure. One also sees that it is much higher for the glass plates than in the case of the alumina targets and that, as anticipated in Sec. II, the fragmentation threshold is much lower in the former case. The large variance values, represented by the error bars, reveal that, for a given pressure, the fluctuations are fairly large. One should note that, although $P_{c}$ has been adjusted to reproduce the average multiplicity, the model also correctly predicts the large variances observed experimentally. This is an intrinsic property of the model.

We now turn to the area distribution and show in Fig. 5, for the alumina plates, the values of $F(A)$, defined as [14]

$$
F(A)=\frac{1}{A} \int_{A}^{A_{0}} n\left(A^{\prime}\right) d A^{\prime},
$$

where $n(A) d A$ is the number of fragments with area between $A$ and $A+d A$. If one focuses on very small sizes, one may assume $A_{0} \rightarrow \infty$ [14]. In this case, if $n(A)$ follows a power law, $F(A)$ would also hold this property.

The dashed line shown in this figure represents the power law fit $F(A) \propto A^{-\beta}$ made in the region of small areas. The results show that the experimental data can be fairly well approximated by this function over about three decades with $\beta=1.1$. This exponent is in agreement with some of the results reported previously $[16,19]$. One observes a steeper drop of $F(A)$ at large areas, which becomes more important as the violence of the impact increases. Nevertheless, the 
fragment distribution associated with not too large fragments $\left(A / A_{0} \lesssim 10^{-2}\right)$ remains essentially unchanged within the pressure range studied in this work.

The steep drop of $F$ at larger fragment sizes has been explained in Ref. [14] as being due to effects associated with a finite size cutoff and $F(s) \propto s^{-\beta} \exp \left(-s / s_{c}\right)$ was used to fit the data, where $s_{c}$ is a characteristic size. We have checked that our experimental results (for both alumina and glass) are not accurately approached by this formula. In Ref. [15], the size distribution of fragments associated with the fragmentation of glass rods were described assuming that $n(A) \propto A^{-\beta}$, but the integration in Eq. (1) has been restricted to the actual size of the rod instead of extending it to infinity. The authors found that their data could be very well reproduced in this way at the lowest dropping heights. However, more violent impacts required the use of two distinct power law regimes, associated with large and small fragments. Following these assumptions one finds [15]

$$
F(A)=\left\{\begin{array}{ll}
F_{<}\left(A / A_{0}\right), & A \leq A_{c} \\
F_{>}\left(A / A_{0}\right), & A \geq A_{c}
\end{array},\right.
$$

where

$$
\begin{aligned}
F_{<}\left(A / A_{0}\right)= & \frac{A_{0}}{A} \frac{a_{1}}{\beta_{1}-1}\left[\left(A / A_{0}\right)^{-\left(\beta_{1}-1\right)}-\left(A_{C} / A_{0}\right)^{-\left(\beta_{1}-1\right)}\right] \\
& +\frac{A_{0}}{A} \frac{a_{2}}{\beta_{2}-1}\left[\left(A_{c} / A_{0}\right)^{-\left(\beta_{2}-1\right)}-1\right],
\end{aligned}
$$

and

$$
F_{>}\left(A / A_{0}\right)=\frac{A_{0}}{A} \frac{a_{2}}{\beta_{2}-1}\left[\left(A / A_{0}\right)^{-\left(\beta_{2}-1\right)}-1\right] .
$$

The crossover point $A_{c}$, as well as $a_{1}, \beta_{1}, a_{2}$, and $\beta_{2}$ are determined through a fit to the experimental values. We found that $A_{c} / A_{0}=0.1,\left(\beta_{1} \approx 0.82, \beta_{2} \approx 1.36\right)$, $\left(\beta_{1} \approx 0.85, \beta_{2} \approx 2.05\right)$, and $\left(\beta_{1} \approx 0.90, \beta_{2} \approx 2.72\right)$, for $P=10,12$, and 14 bar, respectively, give a good description of the data, as depicted by the full lines in the upper panel of Fig. 5. Small deviations are observed near $A / A_{0} \approx 1$, but they stay within the experimental uncertainties in this region. If one adopts this interpretation to the behavior of $F$, the exponent $\beta$ associated with the power law represented by the dashed line in Fig. 5 should be viewed as an apparent exponent since the actual behavior of $F$ in the small area region would be given by $F=f_{1}\left[A / A_{0}\right]^{-\beta_{1}}+f_{2}\left[A / A_{0}\right]^{-\beta_{2}}$, where $f_{1}$ and $f_{2}$ are constants determined from Eq. (3).

In agreement with the results obtained in Ref. [15], we found that $\beta_{1}<\beta_{2}$ and that both exponents increase with the violence of the impact. As also discussed by those authors, the crossover could be interpreted as a switch from lower to higher dimensional fragmentation, although precise conclusions have not been drawn on this respect owing to the inexistence of such a crossover at the lowest impact velocities in their experiment. Our analysis shares other difficulties also remarked in that work as we find $\beta_{1}<\beta_{2}$. This conflicts with the conclusions drawn in Ref. [17] that $\beta$ should increase

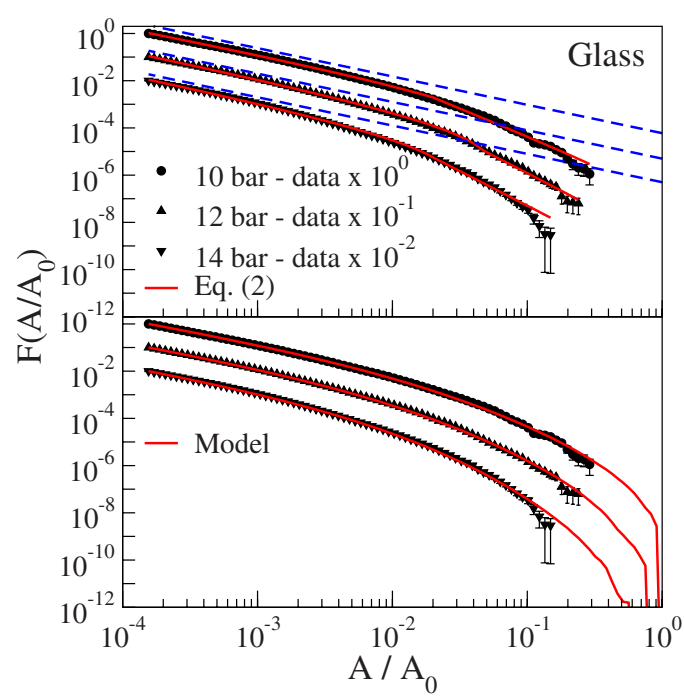

FIG. 6. (Color online) Same as Fig. 5 for glass plates. The dashed line corresponds to a power law whose exponent is $\beta=1.2$. For details, see the text.

with the dimensionality. Therefore, although our data can also be fairly well described by a crossover, an interpretation to this characteristic has yet to emerge.

Qualitatively similar results are observed for the glass plates, as shown in Fig. 6. The exponent of the power law function fitted to the small area region in this case is slightly larger than that obtained for alumina and it corresponds to $\beta=1.2$. The range over which this function remains a good approximation to the actual behavior observed experimentally is considerably smaller than in the case of the alumina plates. The suppression of large areas is much more pronounced in the present case. This is in qualitative agreement with the results shown in Fig. 4, since the fragment multiplicity is much larger in the case of the glass plates than for the alumina ones. The full lines displayed in the upper panel of this figure also correspond to Eq. (2), but the parameters now read $A_{c} / A_{0}=0.02,\left(\beta_{1} \approx 0.99, \beta_{2} \approx 2.36\right)$ and $\left(\beta_{1} \approx 1.09, \beta_{2} \approx 2.92\right)$, where the first set is associated with $P=8$ bar whereas the second one corresponds to $P=10$ and 14 bar. The values of $\beta_{1}$ and $\beta_{2}$ are larger than in the case of the alumina plates, as the area distribution is steeper. The same remarks made in the previous case also hold for the glass plates as the qualitative aspects of the two data sets are very similar.

The lower panels of Figs. 5 and 6 show the predictions of the model described in Sec. III. The agreement of the model results with the experimental data is very good in both cases. Small discrepancies are observed only for large areas, but they are compatible with the experimental uncertainties in this area region. In spite of the success of the model in reproducing the area distribution, its stochastic nature makes it difficult to understand the properties of $F(A)$ just discussed since the model does not allow one to easily make analytical predictions.

As mentioned in Sec. I, it was demonstrated in Ref. [17] that different assumptions for the fragmentation mechanisms may lead to very similar mass distributions. For instance, we consider a very simple picture discussed in Ref. [31] in the 


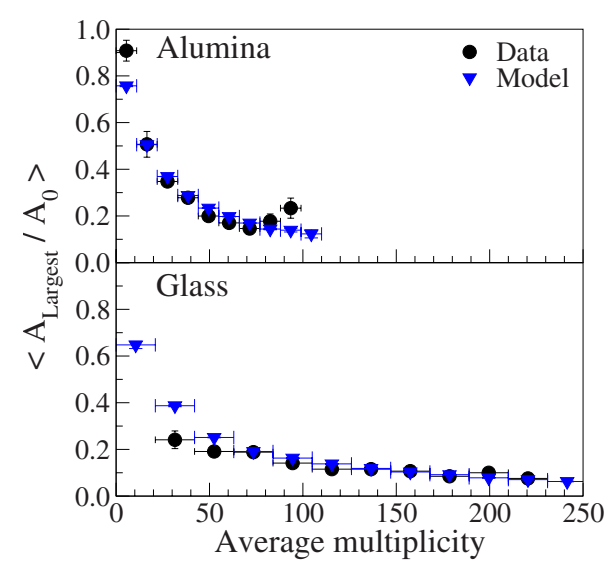

FIG. 7. (Color online) Average value of the largest area within each event as a function of the multiplicity. In the case of the alumina plates, multiplicity bins of 11 units were used whereas 21 units were employed for the glass plates.

framework of one-dimensional fragmentation. If one assumes that rectangular fragments whose longer lengths are equal to the plate's side (which is equal to unity), the area of the $i$ th fragment is given by $A_{i}=l_{i} \times 1$. Its smaller length $l_{i}$ is selected in the following way. If the plate breaks up into $N$ pieces, $l_{1}$ is randomly selected between zero and one. Then, the length $l_{2}$ of the second fragment is uniformly selected between zero and $1-l_{1}$, so that $l_{i}$ is randomly chosen in the interval $\left(0,1-\sum_{j=1}^{i-1} l_{j}\right)$. The probability of observing a fragment of area $A$ is then given by $P(A) \propto A^{-1}$ [31], which furnishes an apparent exponent which is very close to those found above and in previous works. However, the Dalitz plot (see below) for this model, shown in Ref. [31], indicates that the contribution from the largest fragment overwhelms those of all other fragments, in contrast to what is observed in the present work. Furthermore, due to the rather particular shape of the fragments, comparisons with other observables based on the fragments' geometrical properties would rule this model out. Therefore, the description of the size distribution should be regarded as a preliminary selection criterion and additional comparisons with other observables should be made in order to validate any model. In the following, we examine some size correlations in order to seek for further information on the fragmentation process.

The average value of the largest area, $\left\langle A_{\text {Largest }}\right\rangle$, within each event is displayed in Fig. 7 as a function of the fragment multiplicity for the alumina and glass plates. In both cases, $\left\langle A_{\text {Largest }}\right\rangle$ decreases as a function of the fragment multiplicity for not too high multiplicities. In the case of the alumina plates, one observes a slight increase at the highest multiplicity values. This clearly contrasts with the behavior observed in the glass plates. We believe that this deviation is due to the poor statistics of the very high multiplicity events since no anomalies were observed in these events. The predictions of the model agree fairly well with the alumina data for multiplicities smaller than 70-80. They decrease smoothly as a function of the fragment multiplicity. In the case of the glass plates, small discrepancies are observed at the lowest multiplicities, where the model tends to overpredict the size of the largest fragment whereas it underpre-

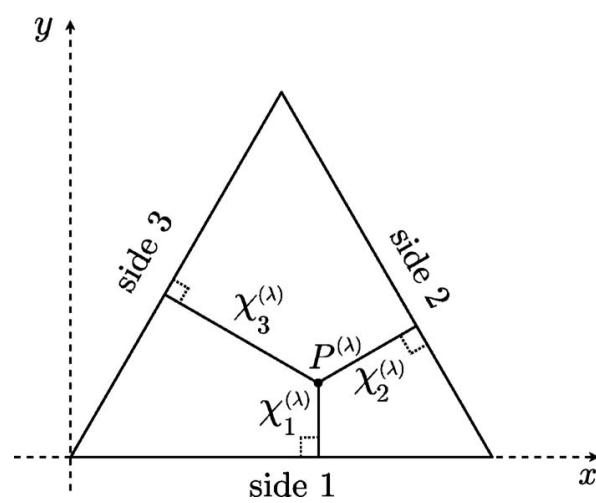

FIG. 8. Illustration of the construction of the Dalitz plot used in this work. For details see the text.

dicts this observable for the alumina plates. This is an indication that, in spite of the very good overall agreement observed above, the details associated with the fragment distribution might not be correctly reproduced by the model.

Important information on the fragmentation pattern may be obtained through the Dalitz plot [38], which is based on the properties of the largest fragments [39]. More specifically, this is constructed by calculating

$$
\chi_{i}^{(\lambda)}=A_{i}^{(\lambda)} / \sum_{k=1}^{3} A_{k}^{(\lambda)}, \quad k=1,2,3,
$$

where $\left\{A_{k}^{(\lambda)}\right\}$ corresponds to the area of the largest three fragments within each event $\lambda$. The quantity $\chi_{i}^{(\lambda)}$ represents the perpendicular distance to the $i$ th side of an equilateral triangle of height 1 , into which a point $P^{(\lambda)}$ associated with a given event $\lambda$ is plotted, as illustrated in Fig. 8. By construction, all the points lie inside the triangle, as $\chi_{1}^{(\lambda)}+\chi_{2}^{(\lambda)}+\chi_{3}^{(\lambda)}$ $=1$. The Cartesian coordinates $\left(x^{(\lambda)}, y^{(\lambda)}\right)$ of the point $P^{(\lambda)}$ associated with $\chi_{1}^{(\lambda)}, \chi_{2}^{(\lambda)}$ and $\chi_{3}^{(\lambda)}$, may be written as $x^{(\lambda)}$ $=\left(\chi_{1}^{(\lambda)}+2 \chi_{3}^{(\lambda)}\right) / \sqrt{3}$ and $y^{(\lambda)}=\chi_{1}^{(\lambda)}$. The indices associated with the triangle's sides are randomized in each event in order to eliminate artificial structures, i.e., the labels assigned to each of the three largest fragments are shuffled and, therefore, we explicitly avoid systematic correlations associated with the fragment sizes. A large concentration of points close to the vertices indicates a fragmentation mode in which the size distribution has a big fragment whereas the others are appreciably smaller. Three big fragments of approximately the same size lead to points grouped around the barycenter of the triangle. Finally, when two fragments have approximately the same size, and are much larger than the remaining ones, one finds points gathered close to the middle point of the triangle's sides.

The experimental Dalitz plot displayed in Fig. 9, obtained in the fragmentation of the alumina plates, shows that there is an important contribution from events in which one fragment is much larger than the others. The results shown in this figure correspond to the cylinder pressure equal to 14 bar, but the conclusion remains valid for the lower pressures employed in this work. The corresponding model results are also displayed on the right side of this figure and the quali- 

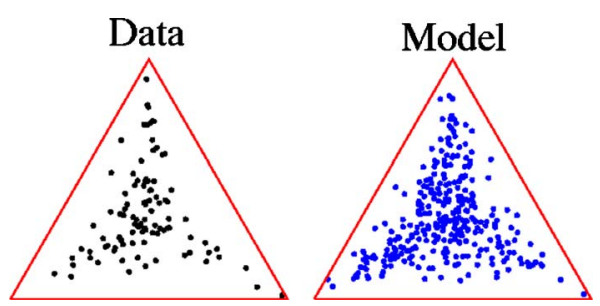

FIG. 9. (Color online) Dalitz plot of the area distribution associated with the fragments produced in the fragmentation of the alumina plates. For details, see the text.

tative agreement with the experimental features is, once more, very good.

A different behavior is observed in the case of the glass plates, for which the points in the Dalitz plot tend to be grouped near the center of the triangle, for all the pressures we considered. As discussed above, this means that the three largest fragments have approximately the same area. Figure 10 shows the corresponding experimental and theoretical plots. Due to the low experimental statistics for this kind of plot, the data associated with different pressures are grouped in this figure, which does not affect our analysis as they are very similar for all pressures. The model correctly predicts the tendency to suppress contributions associated with a dominant fragment. Therefore, the agreement with the data is also fairly good in this case.

Further insight into the properties of the fragment distribution can be obtained by examining the shape parameter $Q$, that we define as

$$
Q=\frac{P^{2}}{4 \pi A},
$$

where $P$ denotes the perimeter of the fragment and $A$ corresponds to its area. Large $Q$ values indicate elongated shapes whereas approximately circular or squared shapes give $Q$ close to unity. In this way, this quantity allows one to obtain information on the shape of the fragments. It is worth mentioning that we analyzed the fractal dimension of the borders and found that it is essentially one-dimensional. Figure 11 displays the average value of $Q$ for different impact velocities, obtained in the fragmentation of the alumina plates. It shows that the small fragments tend to be fairly elongated but this tendency is quickly weakened as the violence of the impact increases. On the other hand, the not too small fragments, i.e., $A / A_{0} \gtrsim 10^{-4}$, are much less elongated than the small ones, for all the pressures studied in this work. The

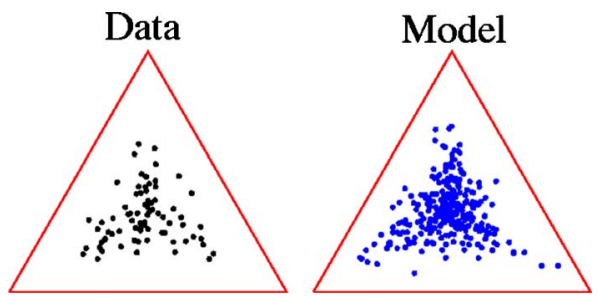

FIG. 10. (Color online) Same as Fig. 9 for the glass plates. For details, see the text.

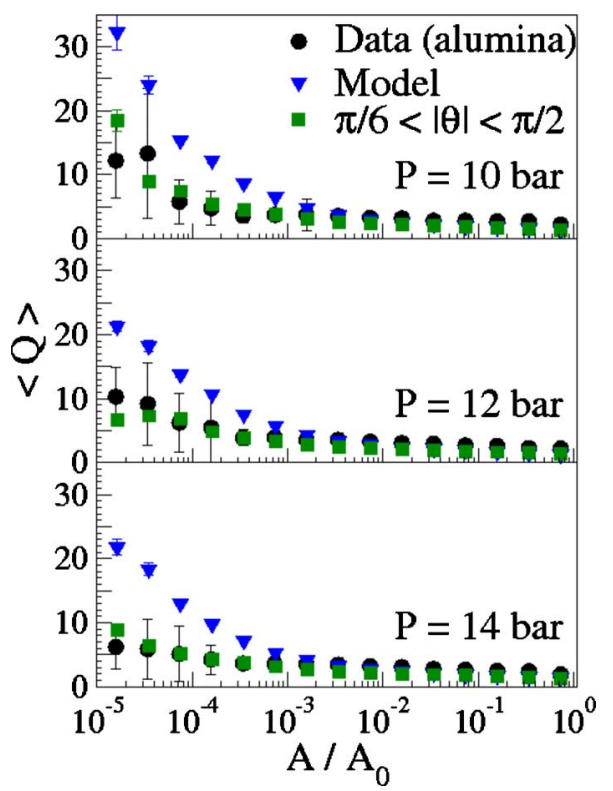

FIG. 11. (Color online) Average $Q$ value versus the fragments' area for different values of the pressure for the fragmentation of the alumina plates. For details, see the text.

understanding of this property requires the development of a model that describes the fracture process accurately. In this context, it should also be interesting to investigate whether there exists a preferential direction to the elongation, but this is beyond the scope of the present study.

The average value of $Q$ obtained with our model is also shown in Fig. 11 and is depicted by the upside-down triangles. The agreement with the experimental values is fairly good for $A / A_{0} \gtrsim 10^{-3}$, but the model predicts too elongated fragments for smaller areas. We come back to this point below.

The fragments produced in the breakup of the glass plates have different properties since the $\langle Q\rangle$ versus $A$ curve is fairly flat, except at the lowest pressures, as shown in Fig. 12. It should be noticed that the vertical scales in Figs. 11 and 12 are not the same. More specifically, the elongation of the fragments originated from the glass plates is much smaller than that observed in the case of the alumina objects. Despite the experimental uncertainties, it is clear that the model, once more, systematically predicts too elongated fragments as already noted in the case of the alumina plates. These results suggest that the observable $Q$ might be a useful tool to selecting models which give an appropriate picture to the fragmentation process.

The tendency to form too elongated fragments in our model can be appreciably reduced by not allowing the cracks initiated at the flaw points to propagate in directions close to the original crack. We have checked that the quality of the agreement with all the observables presented above is maintained if the bifurcation angle $\theta$ is, for instance, restricted to $\pi / 6<|\theta|<\pi / 2$. All the other parameters keep their values, except for $P_{c}$ which is slightly changed (by less than 4\%) in some cases. To illustrate this fact quantitatively, the $Q$ values obtained in this case are depicted by the squares in Figs. 11 and 12. It is clear that the agreement with the data improved considerably. 


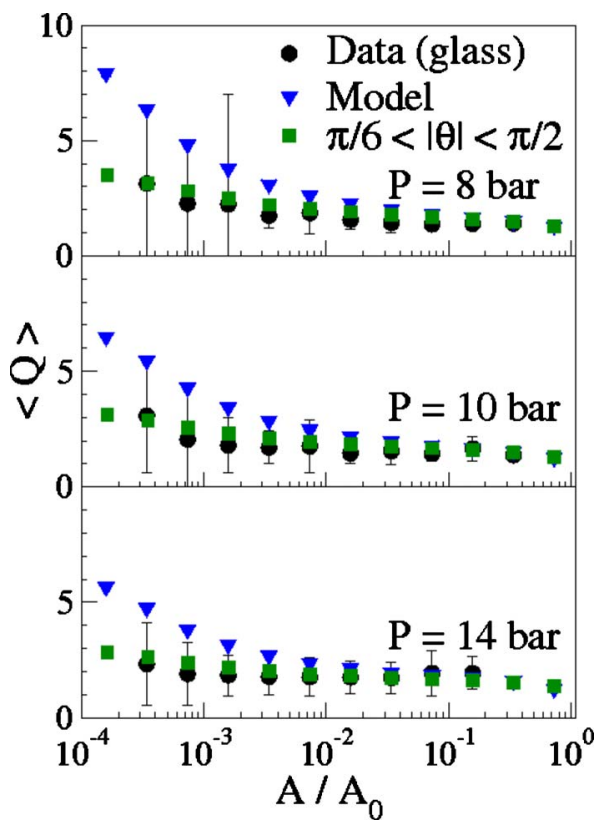

FIG. 12. (Color online) Same as Fig. 11 for the glass plates.

Since there are experimental and theoretical studies $[23,25,40,41]$ that give support to this angular restriction, our results strongly suggest that the new cracks start at relatively large bifurcation angles. We also note that some recent experiments have also found strong evidences for such angle restrictions [42]. Those studies relate them to dynamical instabilities associated with frustrated microcracks initiated from the main one [42-44]. Due to the very schematic character of our model, it is very difficult to speculate on a possible link between it and the picture proposed in those works. As a matter of fact, there is a close resemblance between our model and the scenario investigated experimentally by RaviChandar and Knauss [45], where defects (flaw points) ahead of the tip of the propagating crack can be randomly excited due to the local intense stress field. Those studies also suggest angular restrictions in quantitative agreement with those used in this work. In spite of these encouraging considerations, we preferred not to tune this model parameter and we keep this conclusion on a qualitative level. We postpone detailed discussions on this subject to future work when further observables will be analyzed. In this work we intend to stress that models can reproduce global quantities, such as the mass distribution or the average fragment multiplicity, whereas they can fail in describing more detailed information on the fragments' geometrical properties and that important physical aspects can be revealed by restricting the model parameters.

As a final remark, we should note that the experimental $\langle Q\rangle$ values for small areas are not biased by the fact that we discard fragments whose areas are smaller than $A_{\text {cut }}$. This lower limit is a safe cut which is much larger than the image resolution. The specific values of $A_{\text {cut }}$ have been selected for the alumina and glass plates based on the criterion that the dimensions of the fragments are larger than the thickness of the plates due to the ambiguity associated with the identification of the corresponding dimensions. Although smaller fragments could be identified, they have been excluded from the actual calculation of $\langle Q\rangle$ and other observables. Qualitative analyses of such small fragments indicate that our results should not be impacted by the consideration of these fragments.

\section{CONCLUDING REMARKS}

In summary, we have presented the results of an experiment in which the fragmentation of two different brittle materials due to lateral impact was studied. In agreement with former experimental works, we found that the size distribution is given by an apparent power law over a wide range of sizes. The power law exponents are very similar for both materials, i.e., glass and alumina, and, respectively, correspond to $\beta \approx 1.2$ and $\beta \approx 1.1$, which are close to the values obtained in Refs. $[14,16,17,19]$. We also found that the size distributions are fairly well described by two distinct power laws associated with small and large fragments but, as in Ref. [15], this fact still lacks a theoretical interpretation.

Going beyond those studies, the present experiment also focused on the geometric properties of the fragments. The description of these properties provides strict tests to theoretical models. More specifically, we found that the size distribution and average fragment multiplicities are very well described by the simple fragmentation model presented here, whereas it failed to reproduce the average elongation of the fragments' shapes. This quantity is reproduced by the model only if the bifurcation angle of the fractures is restricted to relatively large angles with respect to the propagation of the initial crack. This is in qualitative agreement with the experimental results reported in Refs. [23,42-45] and the theoretical studies discussed in Refs. [25,40,41]. We believe that careful comparisons of these experimental observables [46] with the predictions of different models might be very useful in helping to establish a clear scenario for the fragmentation process.

\section{ACKNOWLEDGMENTS}

We would like to acknowledge CNPq, CAPES, FAPERJ BBP grants, CNPq-PROSUL, and the joint PRONEX initiative of $\mathrm{CNPq}$ and FAPERJ under Contract No. 26.171.528.2006, for partial financial support. 
[1] D. L. Block, F. Bournaud, F. Combes, R. Groess, P. Barmby, M. L. N. Ashby, G. G. Fazio, M. A. Pahre, and S. P. Willner, Nature (London) 443, 832 (2006).

[2] E. Ryan, Annu. Rev. Earth Planet. Sci. 28, 367 (2000).

[3] E. Bonnet, O. Bour, N. E. Odling, P. Davy, I. Main, P. Cowie, and B. Berkowitz, Rev. Geophys. 39, 347 (2001).

[4] R. Korsnes, S. R. Souza, R. Donangelo, A. Hansen, M. Paczuski, and K. Sneppen, Physica A 331, 291 (2004).

[5] A. Chmel, V. N. Smirnov, and L. V. Panov, Ocean Science 3, 291 (2007)

[6] C. B. Das, S. Das Gupta, W. G. Lynch, A. Z. Mekjian, and M. B. Tsang, Phys. Rep. 406, 1 (2005).

[7] E. E. B. Campbell and F. Rohmund, Rep. Prog. Phys. 63, 1061 (2000).

[8] D. Tilert, G. Svedbojörk, F. Ouchterlony, B. Nilsson, A. Tmun, and L. Mattsson, Int. J. Impact Eng. 34, 1936 (2007).

[9] S. Sadrai, J. Meech, M. Ghomshei, F. Sassani, and D. Tromans, Int. J. Impact Eng. 33, 723 (2006).

[10] F. E. Silva, L. L. Gonçalves, D. B. B. Ferreira, and J. M. A. Rebello, Chaos, Solitons Fractals 26, 481 (2005).

[11] J. Åström, M. Kellomäki, and J. Timonen, Phys. Rev. E 55, 4757 (1997).

[12] L. B. Freund, Dynamic Fracture Mechanics (Cambridge University Press, Cambridge, England, 1989).

[13] W. Weibull, ASME J. Appl. Mech. 18, 293 (1951).

[14] L. Oddershede, P. Dimon, and J. Bohr, Phys. Rev. Lett. 71, 3107 (1993).

[15] E. S. C. Ching, S. L. Lui, and K. Q. Xia, Physica A 287, 83 (2000).

[16] T. Kadono, Phys. Rev. Lett. 78, 1444 (1997).

[17] A. Meibom and I. Balslev, Phys. Rev. Lett. 76, 2492 (1996).

[18] F. Wittel, F. Kun, H. J. Herrmann, and B. H. Kroplin, Phys. Rev. Lett. 93, 035504 (2004).

[19] P. M. C. de Oliveira, C. A. F. Leite, C. V. Chianca, J. S. Martins, and C. F. Moukarzel, Physica A 375, 375 (2007).

[20] O. Sotolongo-Costa, Y. Moreno-Vega, J. J. Lloveras-González, and J. C. Antoranz, Phys. Rev. Lett. 76, 42 (1996).

[21] J. R. Gladden, N. Z. Handzy, A. Belmonte, and E. Villermaux, Phys. Rev. Lett. 94, 035503 (2005).

[22] S. Santucci, K. J. Måløy, A. Delaplace, J. Mathiesen, A. Hansen, Jan Øistein Haavig Bakke, J. Schmittbuhl, L. Vanel, and P. Ray, Phys. Rev. E 75, 016104 (2007).

[23] L. R. Xu and A. J. Rosakis, Exp. Tech. 27, 23 (2003).

[24] D. R. Curran, L. Seaman, and D. A. Schockey, Phys. Rep. 147, 253 (1987).

[25] E. H. Yoffe, Philos. Mag. 42, 739 (1951).

[26] E. Sharon and J. Fineberg, Nature (London) 397, 333 (1999).

[27] H.-J. Tillemans and H. J. Herrmann, Physica A 217, 261 (1995)

[28] H. A. Carmona, F. K. Wittel, F. Kun, and H. J. Herrmann, Phys. Rev. E 77, 051302 (2008).

[29] F. Kun and H. J. Herrmann, Phys. Rev. E 59, 2623 (1999).

[30] B. Behera, F. Kun, S. McNamara1, and H. J. Herrmann, J. Phys.: Condens. Matter 17, S2439 (2005).

[31] F. P. M. dos Santos, R. Donangelo, and S. R. Souza, Physica A 374, 680 (2007).

[32] J. A. Astrom, F. Ouchterlony, R. P. Linna, and J. Timonen, Phys. Rev. Lett. 92, 245506 (2004).

[33] M. Marsili and Y. C. Zhang, Phys. Rev. Lett. 77, 3577 (1996).

[34] G. Hernandez, L. Salinas, and A. Avila, Physica A 370, 565 (2006).

[35] E. S. C. Ching, Y. Y. Yiu, and K. F. Lo, Physica A 265, 119 (1999).

[36] N. Sator, S. Mechkov, and F. Sausset, Europhys. Lett. 81, 44002 (2008).

[37] A. Diehl, H. A. Carmona, L. E. Araripe, J. S. Andrade, and G. A. Farias, Phys. Rev. E 62, 4742 (2000).

[38] R. H. Dalitz, Philos. Mag. 44, 1068 (1953).

[39] P. Kreutz et al., Nucl. Phys. A. 556, 672 (1993).

[40] A. Shukla, H. Nigam, and H. Zervas, Eng. Fract. Mech. 36, 429 (1990).

[41] J. W. Dally, Exp. Mech. 19, 349 (1979).

[42] E. Sharon and J. Fineberg, Phys. Rev. B 54, 7128 (1996).

[43] H. Schäfer, E. Sternin, R. Stannarius, M. Arndt, and F. Kremer, Phys. Rev. Lett. 76, 2177 (1996).

[44] A. Livne, G. Cohen, and J. Fineberg, Phys. Rev. Lett. 94, 224301 (2005).

[45] K. Ravi-Chandar and W. G. Knauss, Int. J. Fract. 26, 65 (1984).

[46] The experimental data presented in this work is available upon request. 